\title{
The effect of smoking on bleeding on probing after nonsurgical periodontal therapy: a quasi-experimental study
}

\author{
Rodrigo ARDAIS \\ Ticiane de Góes MÁRIO \\ Jociana BOLIGON \\ Karla Zanini KANTORSKI \\ Carlos Heitor Cunha MOREIRA
}

Department of Stomatology, Dental School, Universidade Federal de Santa Maria UFSM, Santa Maria, RS, Brazil.

Declaration of Interests: The authors certify that they have no commercial or associative interest that represents a conflict of interest in connection with the manuscript.

\section{Corresponding Author:}

Carlos Heitor Cunha Moreira

E-mail: carlosheitor@rosulonline.com.br

DOI: 10.1590/1807-3107BOR-2014.vol28.0058

Submitted: Mar 21, 2014

Accepted for publication: Jun 24, 2014

Last revision: Sep 22, 2014

\begin{abstract}
The objective of this study was to evaluate the effect of smoking on response to nonsurgical periodontal therapy using the primary outcome measure of bleeding on probing (BoP). An periodontist performed periodontal therapy on 11 smokers and 14 never smokers with periodontitis. Two examiners assessed visible plaque index, gingival bleeding index, probing pocket depth (PPD), BoP, suppuration on probing and clinical attachment level (CAL), at baseline and three months after therapy. BoP was categorized as 0 (absent), 1 (small bleeding point) and 2 (blood flow from the sulcus). Total BoP value was obtained by summing values of 1 and 2 . All subjects had significant reductions in mean PPD and percentages of sites with BoP, with no difference between the groups. Only never smokers presented statistically significant CAL gain. $\mathrm{BoP}$ was significantly and consistently reduced at sites with initial PPDs of 1-3 $\mathrm{mm}$ and 4-6 $\mathrm{mm}$ in both groups. At sites with deep PPD ( $\geq 7 \mathrm{~mm})$, never smokers showed a greater mean reduction in the number of sites with BoP than did smokers $(p<0.05)$. Never smokers had significantly greater reduction in $\mathrm{BoP} 2$ than smokers, at sites with moderate and deep baseline PPDs. The first group had a significant increase of BoP 1, at sites with initial PPDs of 4-6 mm. Thus, periodontal therapy reduced BoP in both groups. However, smoking could negatively affect the BoP reduction at deeper sites after nonsurgical periodontal therapy.
\end{abstract}

Keywords: Periodontitis; Wound Healing; Inflammation; Tobacco Products; Tobacco Use.

\section{Introduction}

Smoking is a well-established risk factor for periodontitis. ${ }^{1,2,3}$ It has been associated with a two- to eight-fold increase in the risk of attachment loss based on disease severity and history of smoking., ${ }^{4,5}$

Previous studies have found that changes in bleeding on probing (BoP) were associated with a worse response to nonsurgical periodontal therapy (NSPT) in smokers. ${ }^{6,7}$ A systematic review ${ }^{8}$ examined the apparent absence of differences in BoP between smokers and nonsmokers, but no meta-analysis was performed due to heterogeneity among included studies. Subsequent clinical trials have presented controversial results, $, 10,11$ and observational studies have been inconclusive due to methodological limitations. ${ }^{12,13}$ Thus, the response of smokers to periodontal therapy as indicated by BoP has not been established. 
The main goals of periodontal treatment are to maintain clinical attachment level (CAL) and resolve inflammation. ${ }^{14}$ Periodontal inflammation has typically been evaluated using BoP. ${ }^{15,16}$ This parameter is associated with the presence of biofilm and subgingival calculus, ${ }^{17}$ and has been used to indicate the need for subgingival intervention. ${ }^{18,19}$

During periodontal maintenance, $\mathrm{BoP}$ and probing pocket depth (PPD) are used as prognostic factors for future periodontal disease activity. ${ }^{20}$ The absence of BoP has been shown to be a good indicator of stable $\mathrm{CAL}$, and its presence has a moderate predictive value for future attachment loss. ${ }^{21}$ Although one point with minimal bleeding at one site does not have the same clinical meaning as more intense bleeding, BoP is considered to be the best parameter for predicting attachment loss. ${ }^{14}$

Despite the importance of BoP in the diagnosis of periodontal inflammation, a limited number of studies have evaluated the effect of smoking on the response to NSPT using this parameter as a primary outcome measure. ${ }^{2}$ Thus, the objective of this study was to compare responses to NSPT between smokers and those who had never smoked (never smoker) using the primary outcome measure of BoP. The primary hypothesis was that smoking would have no effect on BoP.

\section{Methodology}

\section{Study design and sample}

This quasi-experimental study involved subjects with periodontitis ${ }^{22}$ who sought treatment at the dental school of the Universidade Federal de Santa Maria, southern Brazil, between September 2010 and October 2011. Subjects were informed of the purpose of the study and volunteers were interviewed regarding their health and habits. Patients were also clinically screened to determine their eligibility. Eligible subjects provided written informed consent. This study was performed in accordance with the Declaration of Helsinki and was approved by the Ethics Committee in Research of Universidade Federal de Santa Maria, RS, Brazil (CAAE: 0325.0.243.000-10).

Eligible subjects had four teeth with $\mathrm{CAL} \geq 5 \mathrm{~mm}$, PPD $\geq 5 \mathrm{~mm}$, and BoP at one or more sites. Third molars, teeth indicated for extraction and those with endo-periodontal lesions were not evaluated.
The exclusion criteria were: pregnancy, diabetes mellitus, use of anti-inflammatory or antibiotic agents within the previous 6 months, periodontal therapy in the last 12 months, or use of any medications associated with gingival increase. Previous smokers and those who smoked $<10$ cigarettes/day were also excluded from the study. Subjects were classified as smokers ( $\geq 10$ cigarettes/day for $\geq 6$ months) or never smokers.

\section{Sample size}

The sample size was estimated by considering a clinically significant difference of $20 \%$ in the average percentage of sites with BoP between groups, with a standard deviation of $16.7 \%{ }^{10}$ By applying a type I error of 0.05 and a power of 0.80 , we determined that 11 patients per group were required. To compensate for possible losses during the follow-up period, 15 patients were included in each group.

\section{Evaluation}

Demographic, socioeconomic, behavioral, and medical data were collected by interview at baseline. Smoking-related data were collected by self-reporting and standardized interviews at baseline and 3 months after final therapy. Pack/year values were determined by multiplying the number of packs smoked per day by the total number of smoking years.

Clinical parameters were assessed in the following order: visible plaque index (VPI), ${ }^{23}$ gingival bleeding index (GBI), ${ }^{23} \mathrm{PPD}, \mathrm{BoP}$, suppuration on probing (SP) and CAL. Two examiners (TGM and JB) evaluated all parameters at baseline and 3 months after final therapy at six sites (distobuccal, midbuccal, mesiobuccal, mesiolingual, midlingual, and distolingual) per tooth using a manual probe (CP15 UNC; Neumar, São Paulo, Brazil). BoP was evaluated $15 \mathrm{~s}$ after measuring PPD and categorized as 0 (absent), 1 (small bleeding point), or 2 (blood flow from the sulcus); BoP values were modified based on a previous study. ${ }^{24}$ The total BoP value for each patient was obtained by summing all values of 1 and 2. SP was categorized as present or absent.

\section{Measurement reproducibility}

The examiners were trained in the assessment of VPI, GBI, and BoP. Intra- and interexaminer calibration was performed for PPD and CAL using 10 patients by 
duplicating periodontal examinations at a 1-week interval. Reproducibility was tested using the weighted $\pm 1 \mathrm{~mm}$ kappa test. Prior to initiating the study, kappa values for examiners 1 and 2 were 0.97 and 0.96 (PPD), and 0.88 and 0.70 (CAL), respectively; interexaminer kappa values were 0.94 (PPD) and 0.84 (CAL). During the study period, kappa values for examiners 1 and 2 were 0.95 and 0.99 (PPD), and 0.93 and 0.91 (CAL), respectively; interexaminer kappa values were 0.96 (PPD) and 0.86 (CAL).

\section{Nonsurgical periodontal therapy}

After baseline examination, an experienced periodontist (RA) performed NSPT on all patients. This treatment consisted of the removal of all biofilm-retentive factors (supragingival calculus, carious tissue and sealing cavities, excess dental restoration material, teeth requiring extraction), oral hygiene instruction, and scaling and root planing under local anesthesia. After therapy, the subjects were evaluated every 2 weeks for biofilm control over a 3-month period. At each visit, the patients again received oral hygiene instruction and professional removal of supragingival biofilm. No subgingival intervention was performed during this period.

\section{Outcomes}

BoP was the primary outcome. Secondary outcomes were VPI, GBI, PPD, CAL, SP, and BoP values of 1 and 2.

\section{Data analysis}

The analysis was performed by protocol and included only subjects who completed the study. Descriptive analysis of clinical data was performed using averages, standard deviations, and average percentages. PPD was classified as shallow (1-3 mm), moderate (4-6 $\mathrm{mm}$ ), or deep ( $\geq 7 \mathrm{~mm}$ ). Changes in BoP $1, \mathrm{BoP} 2$ and total BoP values were analyzed according to baseline PPD classifications. The normality of data distribution was verified using the Shapiro-Wilk test. Intragroup differences were examined using the paired $t$-test (normal distribution) and Wilcoxon signed-ranks test. Differences between groups were evaluated using the independent $t$-test (normal distribution) and MannWhitney test. Categorical variables were analyzed using the chi-squared test. A $P$ value $<0.05$ was considered statistically significant. All analyses were performed using PASW Statistics 18 software (IBM, Chicago, USA).

\section{Results}

Of 30 subjects initially included in the study, two smokers declined participation and three subjects (two smokers and one never smoker) were lost to follow up (missed regular treatment appointments; Figure 1). No significant difference in baseline clinical and demographic characteristics was observed between subjects who completed the study and those included at baseline (data not shown).

Smokers had consumed a mean of 20 cigarettes (one pack)/day over 25 years, and interviews conducted 3 months after final therapy revealed no change in smoking status in either group. Smokers had more visible biofilm than never smokers $(p=0.03)$. However, GBI values were four times larger among never smokers than among smokers $(p=0.00)$. No significant difference in BoP or PPD was observed between groups. Smokers had a greater prevalence of SP and a higher mean CAL than never smokers (Tables 1 and 2).

A significant reduction in the number of sites with visible plaque was observed during the study period in both groups $(p<0.05)$. Smokers showed no significant change in gingival bleeding during the study. Smokers and never smokers showed

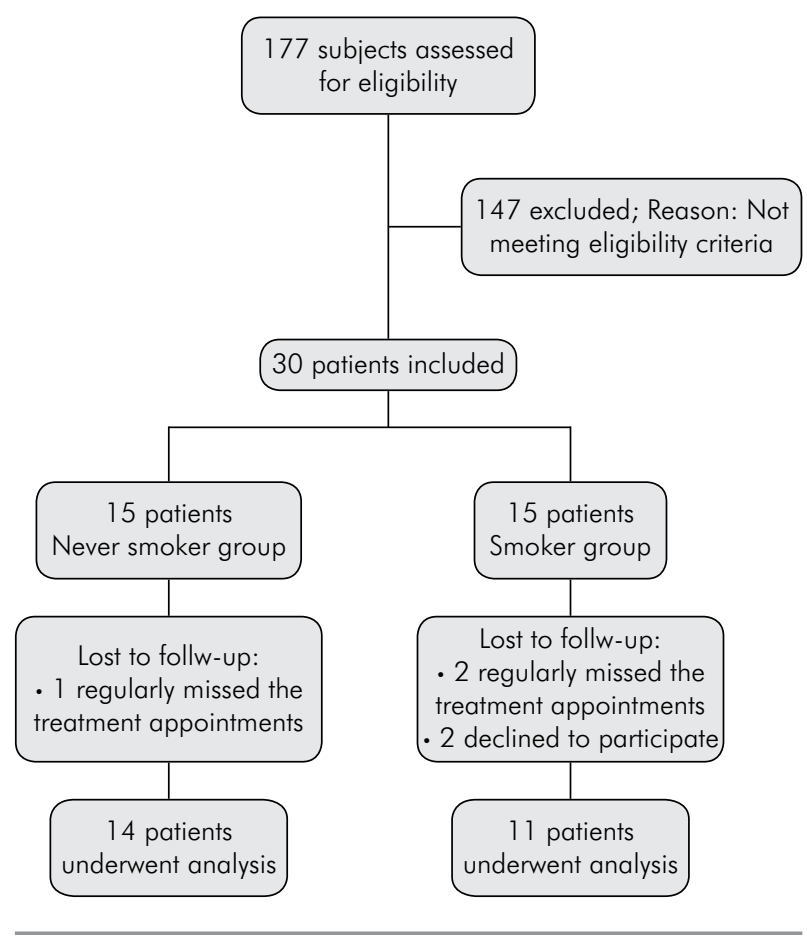

Figure 1. Study flowchart. 
Table 1. Baseline demographical characteristics of the subjects that completed the study's evaluations.

\begin{tabular}{lccc}
\hline & Never smokers $(\mathrm{n}=14)$ & Smokers $(\mathrm{n}=11)$ & $\mathrm{p}$ value \\
\hline Age (years) & $47.64 \pm 10.81$ & $47.00 \pm 7.81$ & 0.87 \\
Gender male \% (n) & $35.70(5)$ & $36.40(4)$ & 0.97 \\
$\mathrm{~N}(\%)$ monthly income $\leq 3$ standard BrS & $64.30(9)$ & $54.50(6)$ & 0.62 \\
$\mathrm{~N}(\%)$ monthly income $>$ 3 standard BrS & $35.70(5)$ & $45.50(5)$ & $\mathrm{NA}$ \\
Cigarettes number /day* & $0 \pm 0$ & $19.27 \pm 3.28$ & $\mathrm{NA}$ \\
Duration of smoking (years)* & $0 \pm 0$ & $25.68 \pm 9.52$ & $\mathrm{NA}$ \\
Pack/years* $^{*}$ & & $25.77 \pm 12.37$ & 0.02 \\
Teeth number* & $25.35 \pm 2.97$ & $21.09 \pm 5.14$ & \\
\hline
\end{tabular}

${ }^{*}$ Mean $( \pm S D)$. Significant $a=5 \%$

$\mathrm{SD}$, standard deviation; BrS, Brazilian salaries (about \$1000); $\mathrm{NA}=$ not applicable

significant reductions in the percentages of sites with PPDs of 4-6 $\mathrm{mm}$ and $\geq 7 \mathrm{~mm}$, as well as significant increases in the percentages of sites with PPDs of 1-3 mm (all $\mathrm{p}<0.05)$, with no difference between groups detected. The percentages of sites with BoP also decreased significantly in both groups, with no difference between them. Only never smokers showed a significant increase in CAL ( $p=0.00$; Table 2).

Table 3 shows changes in BoP according to PPD categories. BoP was significantly and consistently reduced at sites with initial PPDs of 1-3 mm and 4-6 mm in both groups. However, at sites with deep PPD, never smokers showed a greater mean reduction in the number of sites with BoP than did smokers $(-57.14 \pm 51.35$ vs. $7.57 \pm 70.46 ; P<0.05)$. The percentages of sites classified as BoP 2 with moderate and deep baseline PPDs were reduced to a greater degree among never smokers than among smokers. The percentages of sites classified as BoP 1 were reduced for initial PPDs of 1-3 mm and increased for initial PPDs of 4-6 $\mathrm{mm}$ in both groups. However, this difference was significant only at sites with PPDs of 4-6 mm in never smokers.

\section{Discussion}

The results of this study show a significant reduction in the mean percentage of sites with BoP in smokers and never smokers after NSPT, with no significant difference between groups. However, when sites were analyzed according to baseline PPD, the negative effect of smoking on BoP reduction at sites with deep PPD became evident. Additionally, smokers showed less reduction than never smokers in the number of sites classified as BoP 2 with moderate and deep PPDs.

Table 2. Mean $( \pm$ SD) periodontal parameters at baseline and 3 months post-therapy as well as mean changes $(\Delta)$.

\begin{tabular}{lcccccc}
\hline & \multicolumn{3}{c}{ Never smokers $(\mathrm{n}=14)$} & \multicolumn{3}{c}{ Smokers $(\mathrm{n}=11)$} \\
\cline { 2 - 7 } & Baseline & 3 months & $\Delta$ & Baseline & 3 months & $\Delta$ \\
\hline \% sites VPI & $53.20 \pm 24.55$ & $34.99 \pm 22.46$ & $-18.20 \pm 20.34 \dagger^{*}$ & $71.36 \pm 17.04$ & $28.53 \pm 14.92$ & $-42.82 \pm 16.38 \dagger^{*}$ \\
$\%$ sites GBI & $42.82 \pm 16.38 \dagger^{*}$ & $19.14 \pm 12.33$ & $-13.50 \pm 13.03 \dagger^{*}$ & $8.50 \pm 5.95$ & $8.74 \pm 4.74$ & $0.23 \pm 5.88 \dagger$ \\
PPD & $3.26 \pm 0.44$ & $2.37 \pm 0.17$ & $-0.88 \pm 0.37^{*}$ & $3.46 \pm 0.47$ & $2.69 \pm 0.33$ & $-0.77 \pm 0.36^{*}$ \\
$\%$ sites PPD 1-3 mm & $69.02 \pm 9.02$ & $89.66 \pm 5.06$ & $20.64 \pm 7.85^{*}$ & $55.73 \pm 13.18$ & $80.99 \pm 5.06$ & $25.26 \pm 11.99^{*}$ \\
$\%$ sites PPD 4-6 mm & $24.29 \pm 7.17$ & $9.92 \pm 4.99$ & $-14.36 \pm 7.99^{*}$ & $39.16 \pm 13.10$ & $17.16 \pm 7.38$ & $-22.00 \pm 11.8 *$ \\
$\%$ sites PPD $\geq 7 \mathrm{~mm}$ & $6.67 \pm 5.46$ & $0.40 \pm 0.72$ & $-6.27 \pm 5.38^{*}$ & $5.10 \pm 5.47$ & $1.84 \pm 2.62$ & $-3.26 \pm 3.39^{*}$ \\
CAL & $2.95 \pm 1.01$ & $2.53 \pm 0.97$ & $0.41 \pm 0.31^{*}$ & $4.94 \pm 1.53$ & $4.69 \pm 1.58$ & $0.24 \pm 0.33$ \\
$\%$ sites SP & $9.18 \pm 4.88$ & $1.04 \pm 1.24$ & $-8.13 \pm 5.16^{*}$ & $17.11 \pm 11.87$ & $4.09 \pm 4.75$ & $-13.02 \pm 10.95^{*}$ \\
$\%$ sites BoP & $70.89 \pm 13.00$ & $47.07 \pm 16.05$ & $-23.79 \pm 12.93^{*}$ & $66.48 \pm 19.02$ & $50.00 \pm 18.91$ & $-16.47 \pm 15.25^{*}$ \\
$\%$ sites BoP 1 & $29.79 \pm 15.06$ & $29.89 \pm 12.95$ & $0.10 \pm 15.65$ & $18.20 \pm 8.07$ & $16.56 \pm 4.79$ & $-1.63 \pm 9.64$ \\
$\%$ sites BoP 2 & $41.10 \pm 16.08$ & $17.19 \pm 9.24$ & $-23.90 \pm 12.36^{*}$ & $48.27 \pm 22.60$ & $33.43 \pm 17.37$ & $-14.84 \pm 18.40^{*}$ \\
\hline
\end{tabular}

$\mathrm{SD}$, standard deviation

* Statistical difference within groups $(p<0.05)$

†Statistical difference between groups $(p<0.05)$ 
Table 3. Mean ( \pm SD) BoP values according to PPD categories at baseline and 3 months as well as BoP mean alterations $(\Delta)$.

\begin{tabular}{lcccccc}
\hline & \multicolumn{3}{c}{ Never smokers $(\mathrm{n}=14)$} & \multicolumn{3}{c}{ Smokers $(\mathrm{n}=11)$} \\
\cline { 2 - 7 } & Baseline & 3 months & $\Delta$ & Baseline & 3 months & $\Delta$ \\
\hline BoP 1 (PPD 1-3 mm) & $35.33 \pm 19.01$ & $30.03 \pm 14.39$ & $-5.30 \pm 20.08$ & $22.09 \pm 9.65$ & $17.26 \pm 4.83$ & $-4.82 \pm 10.16$ \\
BoP 1 (PPD 4-6 mm) & $13.70 \pm 10.96$ & $28.15 \pm 18.80$ & $14.44 \pm 22.33^{*}$ & $11.14 \pm 11.44$ & $13.50 \pm 9.02$ & $2.36 \pm 14.62$ \\
BoP 1 (PPD $\geq 7 \mathrm{~mm})$ & $0 \pm 0$ & $0 \pm 0$ & $0 \pm 0$ & $1.13 \pm 3.76$ & $4.54 \pm 15.07$ & $3.40 \pm 15.90$ \\
BoP 2 (PPD 1-3 mm) & $27.31 \pm 18.86$ & $14.18 \pm 9.84$ & $-13.12 \pm 14.20^{*}$ & $34.40 \pm 19.98$ & $28.65 \pm 17.51$ & $-5.75 \pm 12.91$ \\
BoP 2 (PPD 4-6 mm) & $80.74 \pm 12.7$ & $43.50 \pm 19.00$ & $-37.24 \pm 17.13^{*} \dagger$ & $74.24 \pm 19.84$ & $58.53 \pm 12.91$ & $-15.71 \pm 23.55 \dagger$ \\
BoP 2 (PPD $\geq 7 \mathrm{~mm})$ & $78.57 \pm 42.58$ & $21.42 \pm 42.58$ & $-57.14 \pm 51.35^{*} \dagger$ & $53.40 \pm 51.26$ & $57.57 \pm 47.93$ & $4.16 \pm 72.33 \dagger$ \\
BoP (PPD 1-3 mm) & $62,65 \pm 17,08$ & $44,22 \pm 17,84$ & $-18.43 \pm 16.09^{*}$ & $56,49 \pm 18,58$ & $45,91 \pm 19,43$ & $-10.58 \pm 13.85^{*}$ \\
BoP (PPD 4-6 mm) & $94,44 \pm 5,54$ & $71,65 \pm 19,43$ & $-22.79 \pm 17.21^{*}$ & $85,39 \pm 13,67$ & $72,03 \pm 13,45$ & $-13.35 \pm 15.62^{*}$ \\
BoP (PPD $\geq 7 \mathrm{~mm})$ & $78,57 \pm 42,58$ & $21,42 \pm 42,58$ & $-57.14 \pm 51.35^{*} \dagger$ & $54,54 \pm 52,22$ & $62,12 \pm 49,49$ & $7.57 \pm 70.46 \dagger$ \\
\hline
\end{tabular}

$\mathrm{SD}$, standard deviation

* Statistical difference within groups $(p<0.05)$

tStatistical difference between groups $(p<0.05)$

The greater biofilm reduction observed after NSPT in smokers than in never smokers may be due to smokers' high mean baseline scores. The reduction in gingivitis was greater among never smokers, likely due to a low prevalence of GBI in smokers at baseline. These findings are in agreement with those of Dietrich et al., ${ }^{25}$ who showed a high suppressive effect of smoking on marginal gingival inflammatory clinical expression.

The current study revealed no difference in PPD reduction between smokers and never smokers; in contrast, Darby et al. ${ }^{9}$ and Preber and Bergstrom ${ }^{7}$ reported smaller reductions in PPD among smokers than among nonsmokers. We also observed no significant difference in CAL gain between groups, in agreement with Labriola et al. ${ }^{8}$ and Wan et al., ${ }^{11}$ who observed no deleterious effect of smoking on CAL gain. Suppuration is a clinical outcome that is difficult to evaluate due to its low prevalence. The mean number of sites with SP at baseline in smokers was almost double that observed in never smokers, which may indicate that smoking results in increased suppuration. NSPT reduced SP significantly in both groups, with no difference between them.

Labriola et al. ${ }^{8}$ performed a systematic review to evaluate the effect of smoking on BoP alterations after NSPT. Most studies included in their review found no difference in BoP between smokers and nonsmokers; however, variability in methods used to evaluate BoP prevented meta-analysis. Other studies $^{12,13}$ presented controversial findings or were inconclusive due to methodological limitations.
Farina et al. ${ }^{26}$ observed that the probability of a site presenting BoP was associated with site-specific and patient-related factors, with higher risk at deeper sites and lower risk for smokers.

In the present study, no significant difference in total BoPreduction was observed between smokers and never smokers ( $\Delta:-16.47 \pm 15.25$ vs. $-23.79 \pm 12.93)$ when bleeding was evaluated for the dichotomy type. Nevertheless, the negative effect of smoking was observed in deeper pockets $(\Delta: 7.57 \pm 70.46 v s$ s. $-57.14 \pm 51.35)$ when sites were classified according to baseline PPD.

In the analysis of BoP using the categories of BoP 1 and 2, the negative effect of smoking was evident in the reduction of the percentages of sites with BoP 2 in moderate and deep pockets. After treatment, approximately $47 \%$ of sites in never smokers still presented BoP (30\% with BoP 1, 17\% with BoP 2). Although the percentage of sites with BoP was similar (about 50\%) among smokers and never smokers, approximately $33 \%$ of sites in smokers presented $\mathrm{BoP}$ 2 and $17 \%$ presented BoP 1 . Thus, at the end of the study period, the majority of BoP in never smokers was classified as BoP 1, whereas the majority of that in smokers was classified as BoP 2. These findings suggest that the inflammation is more severe and difficult to rectify in smokers than in never smokers. Intragroup analysis showed significant reductions in $\mathrm{BoP}$ and BoP 2 in all PPD strata among never smokers, but BoP reductions only at sites with shallow and moderate PPDs among smokers. Thus, these findings support the hypothesis that smoking has a deleterious effect on the resolution of inflammation. ${ }^{8}$ 
The significant increase in the percentage of sites with moderate PPD classified as BoP 1 after therapy in never smokers may be associated with a reduction in inflammation or probing trauma. This hypothesis can be corroborated by the findings that reduced BoP can correspond to the smallest histological inflammation, ${ }^{27}$ and that nonsmokers may be susceptible to major probe penetration in gingival connective tissue. ${ }^{28}$

Quasi-experimental studies are performed when a randomized clinical trial is not possible due to ethical or execution issues. However, these studies require special attention to potentially confounding variables that can affect results due to the absence of randomization. In the present study, the eligibility criteria ensured similarity between groups in baseline parameters that could influence the outcomes of PPD and BoP, and thus a comparable need for periodontal treatment. The inclusion of

\section{References}

1. GelskeySC. Cigarette smoking and periodontitis: methodology to assess the strength of evidence in support of a causal association. Community Dent Oral Epidemiol. 1999 Feb;27(1):16-24.

2. Bergström J. Periodontitis and smoking: an evidence-based appraisal. J Evid Based Dent Pract. 2006 Mar;6(1):33-41.

3. Cesar Neto JB, Rosa EF, Pannuti CM, Romito GA. Smoking and periodontal tissues: a review. Braz Oral Res. 2012 Dec;26 Suppl 1:25-31.

4. Grossi SG, Zambon JJ, Ho AW, Koch G, Dunford RG, Machtei $\mathrm{EE}$, et al. Assessment of risk for periodontal disease. I. Risk indicators for attachment loss. J Periodontol. 1994 Mar;65(3):260-7.

5. Susin C, Oppermann RV, Haugejorden O, Albandar JM. Periodontal attachment loss attributable to cigarette smoking in an urban Brazilian population. J Clin Periodontol. 2004 Nov;31(11):951-8.

6. Preber H, Bergstrom J. Effect of non-surgical treatment on gingival bleeding in smokers and non-smokers. Acta Odontol Scand. 1986 Apr;44(2):85-9.

7. Preber H, Bergstrom J. The effect of non-surgical treatment on periodontal pockets in smokers and non-smokers. J Clin Periodontol. 1986 Apr;13(4):319-23.

8. Labriola A, Needleman I, Moles DR. Systematic review of the effect of smoking on nonsurgical periodontal therapy. Periodontol 2000. 2005;37:124-37.

9. Darby IB, Hodge PJ, Riggio MP, Kinane DF. Clinical and microbiological effect of scaling and root planing in smoker and non-smoker chronic and aggressive periodontitis patients. J Clin Periodontol. 2005 Feb;32(2):200-6. patients with similar subgingival inflammatory periodontal disease in both groups was important to observe the effects of smoking on the evaluated outcomes. One limitation of the present study was the reliance on subjects' self-reports of the number of cigarettes consumed and smoking duration. Future investigations should use physiological measures of smoking duration and exposure.

\section{Conclusions}

NSPT results in clinical improvements in smokers and those who have never smoked. Similar findings were observed for PPD, BoP, and CAL in both of these patient groups. However, smoking had a negative effect on BoP reduction at deeper sites. In addition, smaller reductions in the percentage of severe BoP (BoP 2) were observed in moderate and deep pockets in smoking patients.

10. Apatzidou DA, Riggio MP, Kinane DF. Impact of smoking on the clinical, microbiological and immunological parameters of adult patients with periodontitis. J Clin Periodontol. 2005 Sep;32(9):973-83.

11. Wan CP, Leung WK, Wong MC, Wong RM, Wan P, Lo EC, et al. Effects of smoking on healing response to non-surgical periodontal therapy: a multilevel modelling analysis. J Clin Periodontol. 2009 Mar;36(3):229-39.

12. Farina R, Simonelli A, Rizzi A, Trombelli L. Effect of smoking status on pocket probing depth and bleeding on probing following non-surgical periodontal therapy. Minerva Stomatol. 2010 Jan-Feb;59(1-2):1-12.

13. Nassrawin NA. Effect of smoking on the response to nonsurgical periodontal therapy. East Mediterr Health J. 2010 Feb;16(2):162-5.

14. Claffey N, Polyzois I. Non-surgical therapy. In: Lindhe J, Karring T, Lang N, editors. Clinical Periodontology and Implant Dentistry. Oxford: Blackwell Munksgaard; 2009. p. 767-79.

15. Greenstein G, Caton J, Polson AM. Histologic characteristics associated with bleeding after probing and visual signs of inflammation. J Periodontol. 1981 Aug;52(8):420-5.

16. Armitage G. Clinical periodontal examination. In: Rose L, Mealey B, Genco R, editors. Periodontics: medicine, surgery, and implants. St. Louis: Elsevier Mosby; 2004. p. 134-46.

17. Checchi L, Montevecchi M, Checchi V, Zappulla F. The relationship between bleeding on probing and subgingival deposits. An endoscopical evaluation. Open Dent J. 2009 Jul 28;3:154-60. 
18. Joss A, Adler R, Lang NP. Bleeding on probing. A parameter for monitoring periodontal conditions in clinical practice. J Clin Periodontol. 1994 Jul;21(6):402-8.

19. Serino G, Rosling B, Ramberg P, Socransky SS, Lindhe J. Initial outcome and long-term effect of surgical and nonsurgical treatment of advanced periodontal disease. J Clin Periodontol. 2001 Oct;28(10):910-6.

20. Claffey N, Egelberg J. Clinical indicators of probing attachment loss following initial periodontal treatment in advanced periodontitis patients. J Clin Periodontol. 1995 Sep;22(9):690-6.

21. Lang NP, Adler R, Joss A, Nyman S. Absence of bleeding on probing. An indicator of periodontal stability. J Clin Periodontol. 1990 Nov;17(10):714-21.

22. Tonetti MS, Claffey N, European Workshop in Periodontology group C. Advances in the progression of periodontitis and proposal of definitions of a periodontitis case and disease progression for use in risk factor research. Group C consensus report of the 5th European Workshop in Periodontology. J Clin Periodontol. 2005;32 Suppl 6:210-3.
23. Ainamo J, Bay I. Problems and proposals for recording gingivitis and plaque. Int Dent J. 1975 Dec;25(4):229-35.

24. Lie MA, Timmerman MF, van der Velden U, van der Weijden GA. Evaluation of 2 methods to assess gingival bleeding in smokers and non-smokers in natural and experimental gingivitis. J Clin Periodontol. 1998 Sep;25(9):695-700.

25. Dietrich T, Bernimoulin JP, Glynn RJ. The effect of cigarette smoking on gingival bleeding. J Periodontol. 2004 Jan;75(1):16-22.

26. Farina R, Tomasi C, Trombelli L. The bleeding site: a multilevel analysis of associated factors. J Clin Periodontol. 2013 Aug;40(8):735-42.

27. Engelberger T, Hefti A, Kallenberger A, Rateitschak $\mathrm{KH}$. Correlations among Papilla Bleeding Index, other clinical indices and histologically determined inflammation of gingival papilla. J Clin Periodontol. 1983 Nov;10(6):579-89.

28. Biddle AJ, Palmer RM, Wilson RF, Watts TL. Comparison of the validity of periodontal probing measurements in smokers and non-smokers. J Clin Periodontol. 2001 Aug;28(8):806-12. 